\title{
ARTICLE \\ Kanglexin, a novel anthraquinone compound, protects against myocardial ischemic injury in mice by suppressing NLRP3 and pyroptosis
}

Yu Bian ${ }^{1}$, Xin $\mathrm{Li}^{1}$, Ping Pang ${ }^{1}$, Xue-ling $\mathrm{Hu}^{1}$, Shu-ting Yu${ }^{1}$, Yi-ning $\mathrm{Liu}^{1}$, Xin $\mathrm{Li}^{1}$, Ning Wang ${ }^{1}$, Jin-hui Wang ${ }^{2}$, Wei Xiao ${ }^{3}$, Wei-jie Du ${ }^{1}$ and Bao-feng Yang ${ }^{1}$

Pyroptosis is a form of inflammatory cell death that could be driven by the nucleotide-binding oligomerization domain-like receptor family pyrin domain-containing 3 (NLRP3) inflammasome activation following myocardial infarction (MI). Emerging evidence suggests the therapeutic potential for ameliorating Ml-induced myocardial damages by targeting NLRP3 and pyroptosis. In this study, we investigated the myocardial protection effect of a novel anthraquinone compound (4,5-dihydroxy-7-methyl-9,10anthraquinone-2-ethyl succinate) named Kanglexin (KLX) in vivo and in vitro. Male C57BL/6 mice were pre-treated either with KLX (20, $40 \mathrm{mg} \cdot \mathrm{kg}^{-1}$ per day, intragastric gavage) or vehicle for 7 consecutive days prior to ligation of coronary artery to induce permanent MI. KLX administration dose-dependently reduced myocardial infarct size and lactate dehydrogenase release and improved cardiac function as compared to vehicle-treated mice $24 \mathrm{~h}$ after MI. We found that MI triggered NLRP3 inflammasome activation leading to conversion of interleukin-1 $\beta$ (IL-1 $\beta)$ and IL-18 into their active mature forms in the heart, which could expand the infarct size and drive cardiac dysfunction. We also showed that MI induced pyroptosis, as evidenced by increased DNA fragmentation, mitochondrial swelling, and cell membrane rupture, as well as increased levels of pyroptosis-related proteins, including gasdermin D, N-terminal GSDMD, and cleaved caspase-1. All these detrimental alterations were prevented by KLX. In hypoxia- or lipopolysaccharide (LPS)-treated neonatal mouse ventricular cardiomyocytes, we showed that KLX (10 $\mu \mathrm{M}) \mathrm{decreased}$ the elevated levels of terminal deoxynucleotidyl transferase dUTP nick end labeling- and propidium iodide-positive cells, and pyroptosis-related proteins. We conclude that KLX prevents MI-induced cardiac damages and cardiac dysfunction at least partly through attenuating NLRP3 and subsequent cardiomyocyte pyroptosis, and it is worthy of more rigorous investigations for its potential for alleviating ischemic heart disease.

Keywords: myocardial infarction; anthraquinone; Kanglexin; ischemic injury; NLRP3 inflammasome; pyroptosis

Acta Pharmacologica Sinica (2020) 41:319-326; https://doi.org/10.1038/s41401-019-0307-8

\section{INTRODUCTION}

Myocardial infarction (MI) is one of the leading causes of hospitalization and death globally. Myocardial injury due to $\mathrm{MI}$ is largely due to the loss of damaged cardiomyocytes, which results in reduced cardiac contractility and cardiac dilatation, eventually leading to heart failure (HF) [1]. The current treatment for $\mathrm{MI}$ is percutaneous coronary intervention, which dramatically improves the survival of patients. However, the reperfusion of blood can further accelerate injury to the ischemic heart, and surviving patients are still at risk for developing HF [2]. Therefore, it is of critical importance to develop novel pharmacological therapeutic strategies to prevent cardiomyocyte death and thus limit myocardial injury in patients with MI.

Programmed cell death and necrosis are two major forms of myocardial injury after MI. In contrast to necrosis, programmed cell death mainly occurs in the infarct border zone and has been identified as an important determinant of the severity of cardiomyocyte injury and the subsequent outcome of MI patients [3]. Recently, a new form of inflammatory programmed cell death known as pyroptosis was recognized. Pyroptosis, which shares the morphological characteristics of apoptosis (typical programmed cell death) and necrosis, including DNA fragmentation, cell swelling, an impairment of membrane integrity, and the release of proinflammatory cytokines such as interleukin-1 $\beta$ (IL-1 $\beta$ ) and IL-18, is initiated by caspase-1 [4]. Specifically, caspase- 1 activation cleaves gasdermin D (GSDMD) and generates $\mathrm{N}$-terminal fragment oligomers within the cell membrane, leading to membrane rupture and pyroptotic cell death through the formation of large pores [5]. Several studies have confirmed the involvement of pyroptosis in response to different pathological stresses, particularly in the postMI heart [6, 7]. The nucleotide-binding oligomerization domain-like receptor family pyrin domain-containing 3 (NLRP3) inflammasome is activated upon $\mathrm{Ml}$ and acts as an initiator of pyroptosis [8]. The activated NLRP3 inflammasome aggravates myocardial injury by

\footnotetext{
${ }^{1}$ Department of Pharmacology (State-Province Key Laboratories of Biomedicine Pharmaceutics of China, Key Laboratory of Cardiovascular research, Ministry of Education), College of Pharmacy, Harbin Medical University, Harbin 150081, China; ${ }^{2}$ Department of Medicinal Chemistry and Natural Medicine Chemistry, Harbin Medical University, Harbin 150081, China and ${ }^{3}$ Jiangsu Kanion Pharmaceutical Co., Ltd., Lianyungang 222001, China

Correspondence: Bao-Feng Yang (yangbf@ems.hrbmu.edu.cn) or Wei-Jie Du (adugo@163.com) or Wei Xiao (xw@kanion.com)
}

Received: 1 June 2019 Accepted: 6 September 2019

Published online: 23 October 2019 
directly activating caspase-1-mediated cell pyroptosis and indirectly through the release of pro-inflammatory cytokines [9]. In contrast, the inhibition of the NLRP3 inflammasome signaling pathway reduces infarct size and preserves cardiac function via attenuating cardiomyocyte pyroptosis in post-MI mice [10-12]. These findings suggest that targeting NLRP3 inflammasome activation and subsequent pyroptosis may be a promising therapeutic strategy for suppressing Ml-induced myocardial injuries.

There is increasing evidence that monomeric compounds with various pharmacological properties can have beneficial effects on cardiovascular and cerebrovascular diseases. In particular, anthraquinones are a crucial class of new and synthetic compounds that possess a broad range of applications. Numerous studies have documented that several anthraquinone compounds provide protection from a variety of cardiac disorders [13-17].

In this study, we chemically designed and synthesized a novel anthraquinone compound (4,5-dihydroxy-7-methyl-9,10anthraquinone-2-ethyl succinate) called Kanglexin (KLX). We aimed to investigate the potential cytoprotective effects of this novel compound against ischemic injury in a mouse model of $\mathrm{MI}$ and the modification of the NLRP3 inflammasome and pyroptosis as underlying cellular and molecular mechanisms.

\section{MATERIALS AND METHODS}

Animals

Animal experiments were performed in accordance with the NIH guide for the Care and Use of Laboratory Animals and were approved by the Animal Ethical Committee of Harbin Medical University. Male C57BL/6 mice ( $~ 8$ weeks old, weighing $20 \pm 2 \mathrm{~g}$ ) were obtained from Changsheng Biotechnology Company (Liaoning, China).

Mouse MI model and drug administration

The mice were randomly divided into four groups of equal numbers (Fig. 1b): the sham group, the $\mathrm{MI}+$ vehicle group, the $\mathrm{MI}+\mathrm{KLX}$-Low (KLX-L; $20 \mathrm{mg} \cdot \mathrm{kg}^{-1}$ ) group, and the MI + KLX-High $\left(\mathrm{KLX}-\mathrm{H} ; 40 \mathrm{mg} \cdot \mathrm{kg}^{-1}\right)$ group. The mice were administered 20 or $40 \mathrm{mg} \cdot \mathrm{kg}^{-1}$ per day KLX by intragastric gavage, and the sham and $\mathrm{Ml}$ groups received an equivalent volume of solvent for 7 days prior to MI surgery. The mouse model of MI was generated using previously described procedures [18]. Briefly, the mice were anesthetized with avertine $\left(0.2 \mathrm{~g} \cdot \mathrm{kg}^{-1}\right.$; Sigma-Aldrich Corporation, USA), and they underwent open-chest surgery to expose the hearts. MI was established by ligation of the left anterior descending coronary artery $2 \mathrm{~mm}$ below the left auricle with a $7 / 0$ nylon suture for $24 \mathrm{~h}$. The surgical procedures for the sham control mice were identical to those for the MI model, but did not include coronary artery ligation. Cardiac function was determined by echocardiographic measurements after anesthesia, and blood and heart tissue were stored at $-80^{\circ} \mathrm{C}$ for later use.

Echocardiographic measurements

Twenty-four hours after surgery, echocardiography was performed using an echocardiographic system with a Vevo2100 ultrasound machine (Visualsonics, Toronto, Canada) at a probe frequency of 10 $\mathrm{MHz}$. The mice were anesthetized with avertine throughout the entire surgery period for noninvasive examination. Two-dimensional images were obtained at the papillary muscles. The ejection fraction (EF) and fractional shortening (FS) were measured on the M-mode a<smiles>CCOC(=O)CCC(=O)Oc1cc(O)c2c(c1)C(=O)c1cc(C)cc(O)c1C2=O</smiles>

d
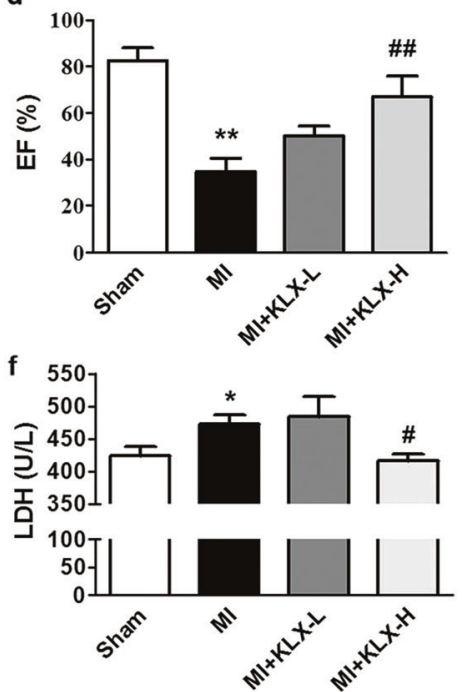

b

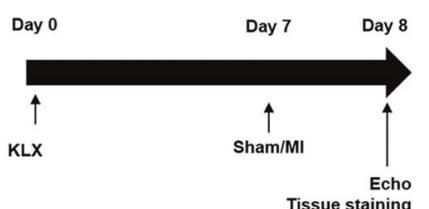

e

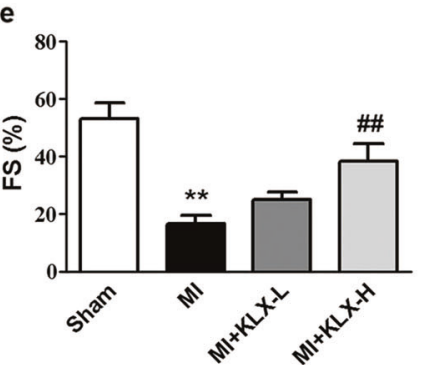

g

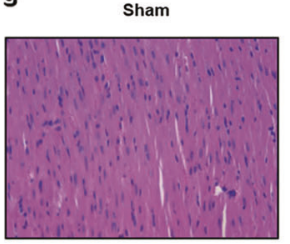

C
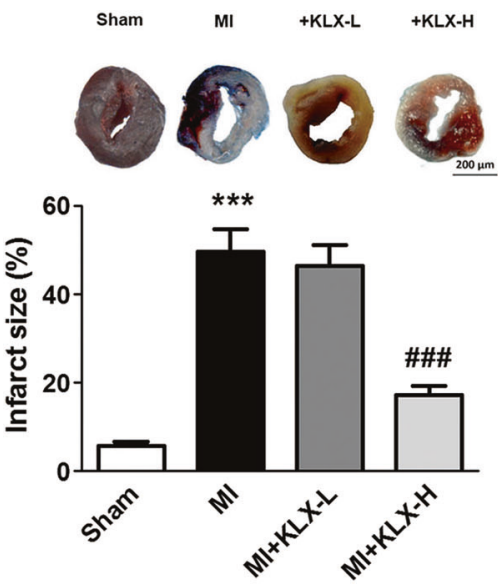

M!
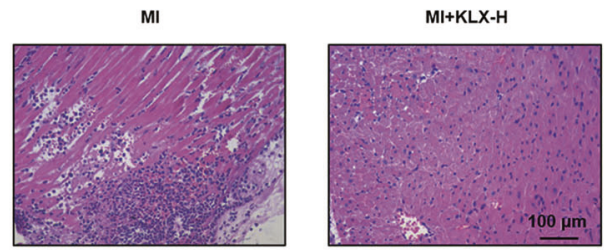

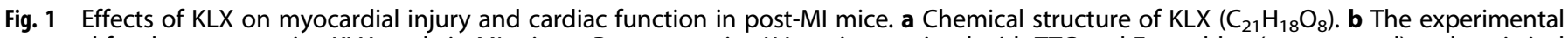
protocol for the preventative KLX study in MI mice. c Representative LV sections stained with TTC and Evans blue (upper panel) and statistical data of the LV infarct size. The infarct size (\%) is expressed as the percentage of infarct area relative to the total LV area. The blue area represents the nonischemic region, the area at risk is stained red, and the infarct region is stained white. Scale bar $=200 \mu \mathrm{m} ; n=5$. KLX-L: $20 \mathrm{mg} \cdot \mathrm{kg}^{-1} ; \mathrm{KLX}-\mathrm{H}: 40 \mathrm{mg} \cdot \mathrm{kg}^{-1}$. d, e Echocardiographic analysis of the LV EF\% and FS\% in the mice; $n=7-8$. f Serum LDH level in the mice; $n=5$. g Representative H\&E staining of paraffin sections of the LV from the mice $(\times 200)$. Scale bar $=100 \mu \mathrm{m}$. The data are presented as the mean \pm SEM; ${ }^{*} P<0.05,{ }^{* *} P<0.01,{ }^{* * *} P<0.001$ vs. sham; ${ }^{\#} P<0.05$, ${ }^{\# \#} P<0.01,{ }^{\# \# \#} P<0.001$ vs. MI 
tracings and calculated by the machine as the average of three cardiac cycles.

\section{Measurement of myocardial infarct size}

The mice were injected through the abdominal aorta with Evans blue (1\%; Solarbio, China) to stain the non-infarcted areas. The hearts were cut transversely into $1-\mathrm{mm}$ sections and stained with $2 \%$ triphenyltetrazolium chloride (TTC; Solarbio) at $37^{\circ} \mathrm{C}$ for 15 min. Images were captured with a microscope (ZEISS, Germany). The infarct size was calculated as the percentage of infarct area relative to the total area of the left ventricle.

\section{Electron microscopic examination}

Cardiac slices were fixed in $2.5 \%$ glutaraldehyde and rinsed with $0.1 \mathrm{~mol} \cdot \mathrm{L}^{-1}$ phosphate-buffered saline (PBS). The samples were placed in $1 \%$ osmium tetroxide at $4{ }^{\circ} \mathrm{C}$. After fixation for $4 \mathrm{~h}$, the samples were stained with $1 \%$ uranyl acetate dehydrated in ethanol and embedded in epoxy resin, and stained with uranyl acetate and lead citrate. The samples were examined under a JEM1200 electron microscope (FEl, USA).

Lactate dehydrogenase release assay

Blood samples were collected before the mice were sacrificed, and the serum was separated. The concentration of lactate dehydrogenase (LDH) in the serum was measured according to the manufacturer's experimental procedure (Nanjing Jiancheng, Jiangsu, China).

\section{Hematoxylin and eosin staining}

Middle-transverse sections of the left ventricular (LV) wall were fixed in $4 \%$ paraformaldehyde for embedding in paraffin. The tissues were cut into 4- $\mu \mathrm{m}$-thick sections and stained with hematoxylin and eosin (H\&E). Images were captured by microscopy (FV300).

Culture and treatment of neonatal mouse ventricular cardiomyocytes

One- to three-day-old neonatal C57BL/6 mice were subjected to open-chest surgery to expose the hearts. The heart tissues were digested into single cardiomyocytes with $0.25 \%$ trypsin (Solarbio, China). The cells were seeded into culture dishes at $1 \times 10^{6}$ cells per well and then exposed to various treatments. For LPS treatment, cardiomyocytes were treated with $1 \mu \mathrm{g} \cdot \mathrm{mL}^{-1}$ LPS for $12 \mathrm{~h}$. For hypoxia treatment, cardiomyocytes were placed in an anoxic chamber with $5 \% \mathrm{CO}_{2}$ and $94 \% \mathrm{~N}_{2}$ for $24 \mathrm{~h}$ and cotreated with $\mathrm{KLX}$ or $0.1 \%$ dimethyl sulfoxide (DMSO) as a vehicle control.

\section{TUNEL and Hoechst/PI staining}

Cardiomyocytes were cultured in 24-well plates at a density of $1 \times$ $10^{5}$ cells per well to evaluate pyroptosis after drug treatment. The cardiomyocytes were fixed and permeabilized in $4 \%$ paraformaldehyde and $0.1 \%$ Triton X-100. The cells were incubated with terminal deoxynucleotidyl transferase dUTP nick end labeling (TUNEL) staining agents at $37^{\circ} \mathrm{C}$ in the dark for $1 \mathrm{~h}$ and DAPI $\left(4^{\prime}, 6-\right.$ diamidino-2-phenylindole) for $15 \mathrm{~min}$. The cardiomyocytes were then incubated with Hoechst 33342 and propidium iodide (PI) (Solarbio, China) in the dark at $4{ }^{\circ} \mathrm{C}$ for $20 \mathrm{~min}$. The fluorescence was detected by confocal laser scanning microscopy (FV300, Olympus, Japan).

Western blot analysis

Protein samples were extracted using lysis buffer containing 1\% protease inhibitor (Roche, Switzerland). Protein concentrations were measured by a BCA protein kit (Beyotime Institute of Biotechnology, Shanghai, China). The proteins were separated by $12 \%$ sodium dodecyl sulfate-polyacrylamide gel electrophoresis and transferred onto polyvinylidene difluoride membranes (PALL, USA). The membranes were incubated with the antibodies against
NLRP3 (1:1000; \#ab97051, Abcam, Cambridge, UK), GSDMD (1:500; \#ab209845, Abcam), IL-1 $\beta$ (1:500; \#ab9722), caspase-1 (1:500; \#WL02996a, Wanleibio, China), IL-18 (1:500; \#A1115, Abclonal, Wuhan, China), and $\beta$-actin (1:1000; \#bs-0061R, BIOSS, Boston, MA, USA) at $4{ }^{\circ} \mathrm{C}$ overnight and then with a fluorescence-conjugated anti-rabbit IgG secondary antibody (1:10,000; LI-COR Bioscience, Lincoln, NE, USA) for 50 min in the dark. The gray value of each protein was quantified by an Infrared Fluorescence Imaging Detector (LI-COR Bioscience) and normalized to $\beta$-actin as an internal control.

RNA isolation and real-time PCR

Total RNA was extracted from myocardial tissues or neonatal mouse ventricular cardiomyocytes (NMVCs) using Trizol reagent (Invitrogen, Carlsbad, CA, USA) according to the manufacturer's instructions. A total of $1 \mu \mathrm{g}$ of RNA was reverse transcribed into complementary DNA under the conditions of $37^{\circ} \mathrm{C}$ for $15 \mathrm{~min}, 98^{\circ} \mathrm{C}$ for $5 \mathrm{~min}$, and $4^{\circ}$ C using a Reverse Transcription kit (Toyobo, Osaka, Japan). SYBR Green (Toyobo) was used for real-time PCR to quantify messenger RNA (mRNA) level with $\beta$-actin as an internal control on a 7500 FAST Real-Time PCR System (ABI, Waltham, MA, USA). The normalized level of NLRP3 (forward primer: 5'-CAACCTCACGTCACACTGCT-3'; reverse primer: 5'-TाTCAGACAACCCCAGGTTC-3') mRNA expression was calculated according to the $2^{-\triangle \Delta \mathrm{Ct}}$ method.

Statistical analysis

The data are presented as the mean \pm SEM. The results were analyzed using Student's $t$ test for two-group comparisons and one-way analysis of variance followed by Tukey's post hoc correction multiple-group comparisons. A $P$ value of $<0.05$ was considered statistically significant. GraphPad Prism 5 was used for the statistical analysis.

\section{RESULTS}

KLX treatment reduces myocardial injury in MI mice

To determine whether KLX (Fig. 1a) possesses cytoprotective effects against MI damage and associated cardiac dysfunction, we employed a mouse model of Ml. The experimental protocol for the in vivo study is depicted in Fig. 1b. Evans blue/TTC double staining was performed to measure the infarct size $24 \mathrm{~h}$ after MI. As shown in Fig. 1c, the infarct size was significantly reduced by treatment with $\mathrm{KLX}-\mathrm{H}$ at a dosage of $40 \mathrm{mg} \cdot \mathrm{kg}^{-1}$ per day. Echocardiographic measurements of LV systolic function showed that, compared to sham operation, $\mathrm{MI}$ leds to dramatic decreases in the EF percentage (EF\%) and FS percentage (FS\%), which were remarkably reversed by $\mathrm{KLX}-\mathrm{H}$ treatment (Fig. $1 \mathrm{~d}$, e). KLX-H failed to alter cardiac function, indicating that this dose of KLX had no toxic effect on the heart (Supplementary Fig. 1). Furthermore, KLX-H remarkedly diminished the release of $\mathrm{LDH}$, an indicator of cardiac damage, from the infarcted myocardium into the serum (Fig. 1f). By comparison, $\mathrm{KLX}$ group at a dosage of $20 \mathrm{mg} \cdot \mathrm{kg}^{-1}$ per day did not elicit any significant beneficial effects (Fig. 1c-f). H\&E staining of LV sections showed a significant reduction in the infiltration of inflammatory cells in the mice treated with KLX-H compared with untreated MI mice (Fig. 1g).

KLX effectively inhibits pyroptosis processes in the mouse hearts with MI

Pyroptosis is a new form of inflammatory programmed cell death, which has been shown to contribute to cardiomyocyte loss in the heart following $\mathrm{MI}[10,12]$. We sought to explore whether the protective effects of KLX on ischemic hearts occur through the attenuation of pyroptosis. TUNEL staining and electron microscopy were used to evaluate the morphological characteristics of pyroptotic cell death in our experimental model. As shown in Fig. 2a, b, Ml caused enormous DNA fragments, mitochondrial swelling, cytoplasmic membrane pore 
a sham

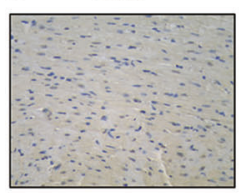

b sham

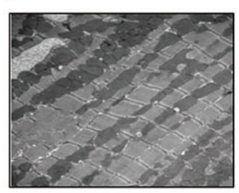

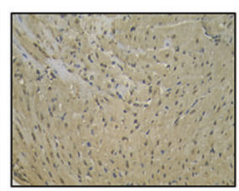

MI

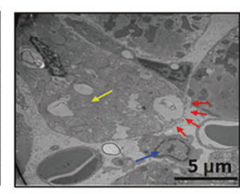

e

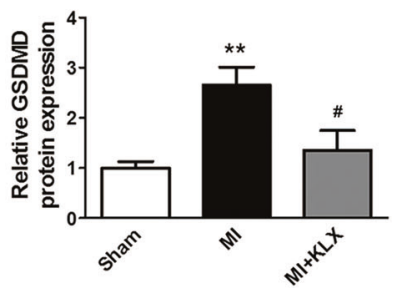

f

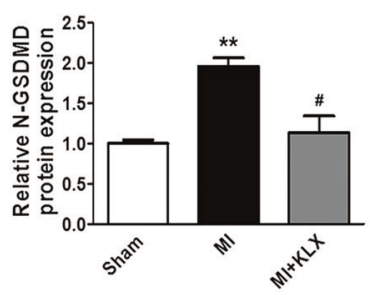

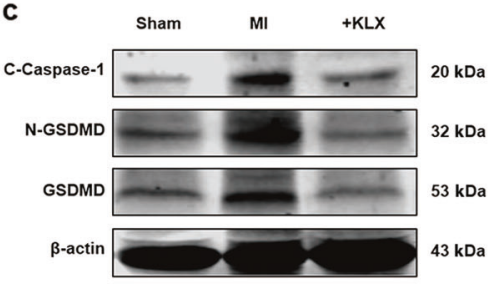

g

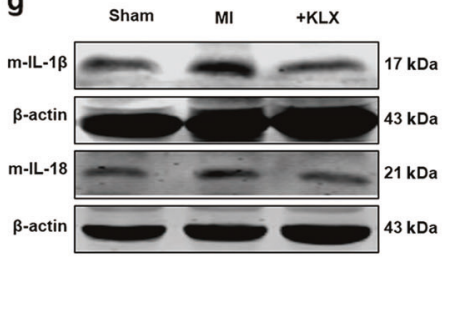

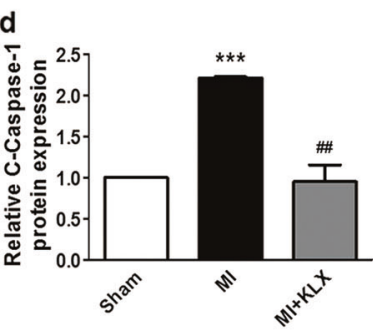

h

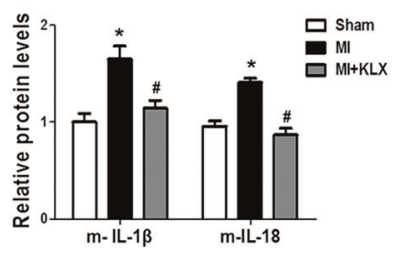

Fig. 2 Effects of KLX on pyroptosis in MI mice. a Representative TUNEL staining of LV sections $(\times 200)$ from the mice. Scale bar $=100 \mu \mathrm{m}$. $\mathbf{b}$ Representative electron micrographs $(\times 10,000)$ of LV sections. The red arrowheads indicate membrane pores, the yellow arrowheads indicate swollen mitochondria, and the blue arrowheads indicate inflammatory cells. c Western blot analysis of cleaved caspase-1, N-GSDMD, and GSDMD in mouse hearts. The relative protein levels of $\mathbf{d}$ cleaved caspase- $1(n=3)$, e GSDMD, and $\mathbf{f}$ N-GSDMD $(n=5)$. $\mathbf{g}, \mathbf{h}$ The relative protein levels of mature IL-1 $\beta(n=5)$ and IL-18 $(n=6)$ in mouse hearts. Protein expressions were normalized to $\beta$-actin. The data are presented as the mean \pm SEM. ${ }^{*} P<0.05$, ${ }^{* *} P<0.01,{ }^{* *} P<0.001$ vs. sham; ${ }^{\#} P<0.05,{ }^{\# \#} P<0.01$ vs. MI. d-f, h One-way ANOVA followed by Tukey's post hoc analysis

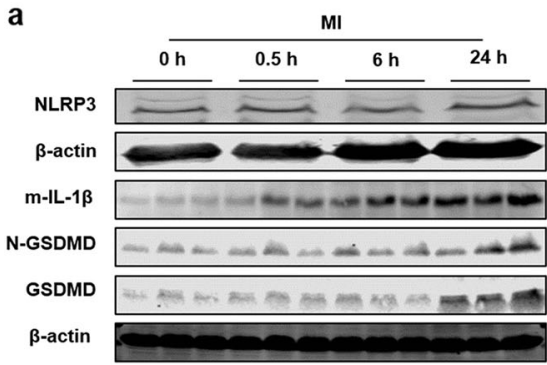

c

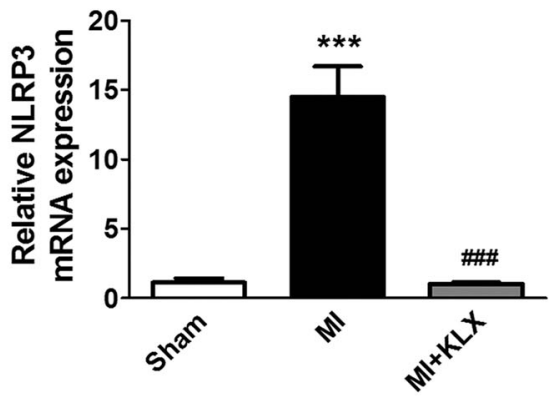

b

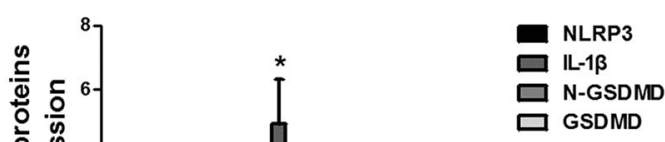

Fig. 3 Effects of KLX on the NLRP3 inflammasome in MI mice. $\mathbf{a}, \mathbf{b}$ Western blot analysis of the protein levels of NLRP3 ( $n=5$ ), mature IL-1 $\beta$ $(n=5), \operatorname{GSDMD}(n=4)$, and N-GSDMD $(n=4)$ at varying time points after MI. The $0 \mathrm{~h}$ time point indicates the sham operation group. C Quantitation of NLRP3 mRNA level $24 \mathrm{~h}$ after MI upon treatment with KLX-H, as determined by qRT-PCR; $n=5$. d Western blot analysis of NLRP3 protein level $24 \mathrm{~h}$ after MI upon treatment with $\mathrm{KLX}-\mathrm{H} ; n=6$. The data are presented as the mean \pm SEM. ${ }^{*} P<0.05,{ }^{* * *} P<0.001$ vs. sham, $\# \# P<0.001$ vs. MI. b. Two-tailed unpaired Student's $t$ test. d One-way ANOVA followed by Tukey's post hoc analysis 
a

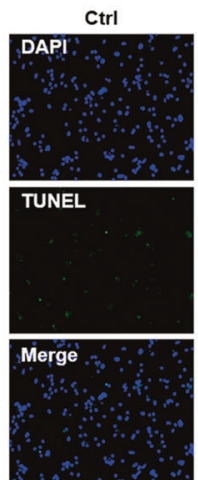

b

Ctrl

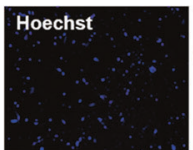

PI

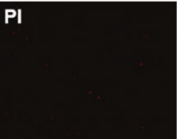

Merge

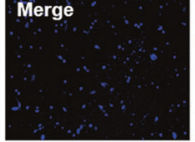

Hypoxia

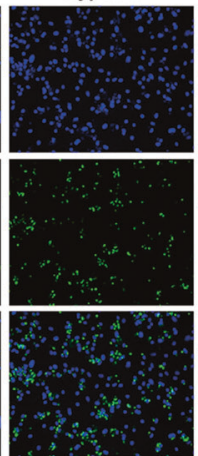

Hypoxia

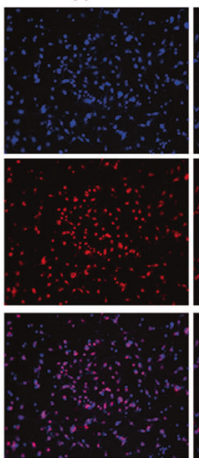

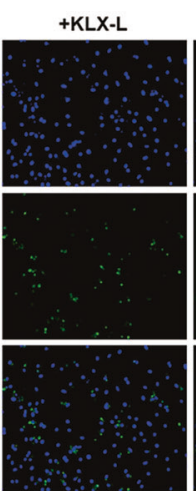

$+K L X-L$

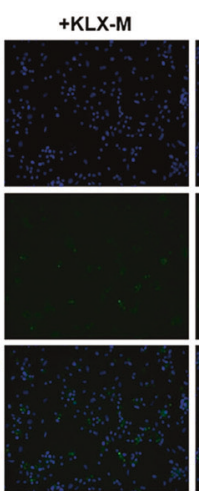

+ KLX-M

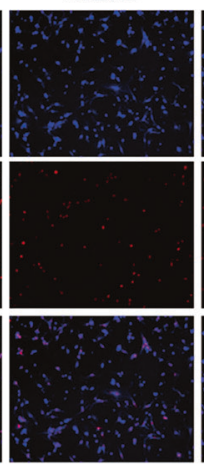

+ KLX-H

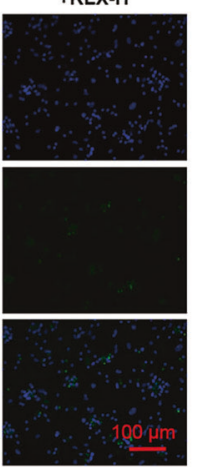

$+\mathrm{KLX}-\mathrm{H}$

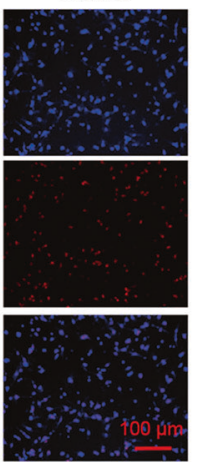

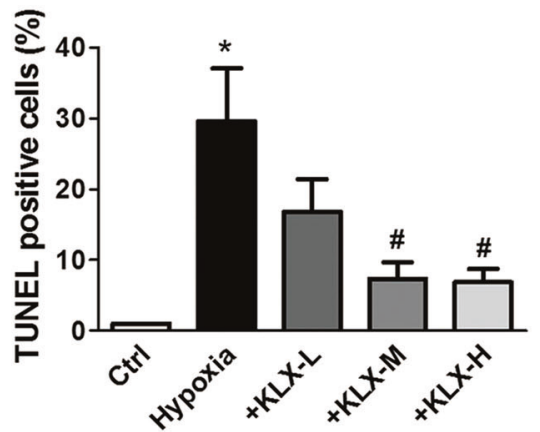

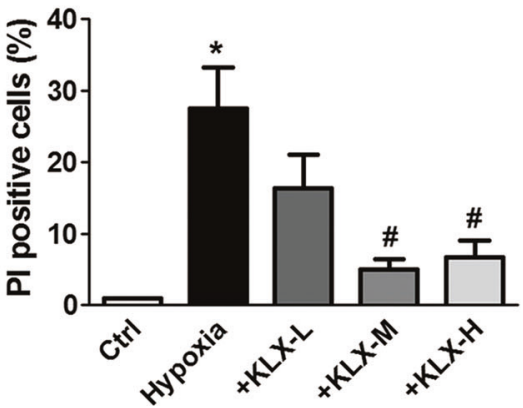

C
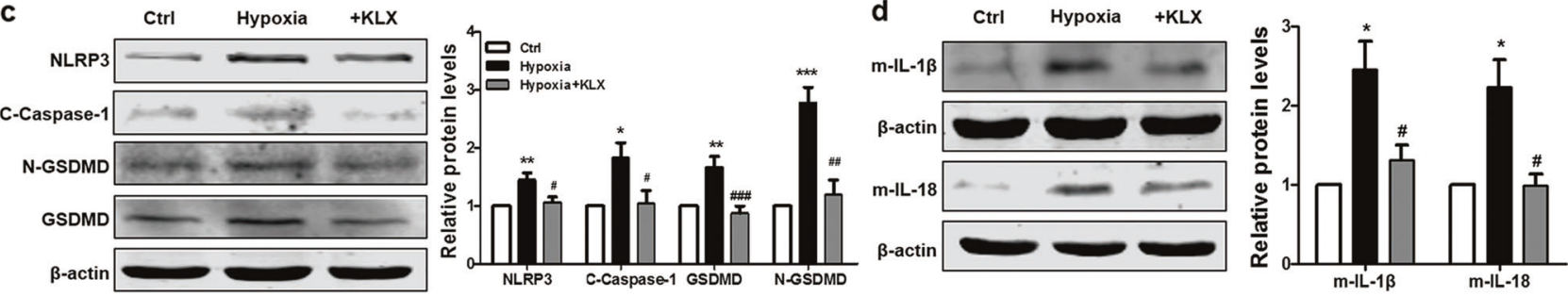

Fig. 4 Effects of KLX on hypoxia-induced NLRP3 activation and pyroptosis in NMVCs. a Representative images of TUNEL staining (left) and the quantitative analysis of TUNEL-positive NMVCs (right). The NMVCs were treated with varying concentrations of KLX (KLX-L: $5 \mu$ M; KLX-M: 10 $\mu \mathrm{M}$; KLX-H: $20 \mu \mathrm{M}$ ) and exposed to hypoxic conditions for $24 \mathrm{~h}$. The nuclei were stained with DAPI (blue), and cells with chromosomal DNA fragmentation were stained with TUNEL (green). Scale bar $=100 \mu \mathrm{m} ; n=4$. b Representative images of Hoechst $33342 / \mathrm{PI}$ staining (left) and the quantitative analysis of PI-positive NMVCs (right). The nuclei were stained with Hoechst 33342, and cells with a ruptured membrane were stained with PI (red). Scale bar $=100 \mu \mathrm{m} ; n=3$. c, d Western blot analysis of the protein levels of NLRP3 $(n=4)$, cleaved caspase- 1 ( $n=5$ ), GSDMD $(n=5)$, N-GSDMD $(n=5)$, mature IL-1 $\beta(n=6)$, and IL-18 $(n=5)$ in NMVCs treated with $10 \mu M$ KLX and exposed to hypoxic conditions for $24 \mathrm{~h}$. The data are presented as the mean \pm SEM. ${ }^{*} P<0.05,{ }^{* *} P<0.01$, ${ }^{* * *} P<0.001 \quad$ vs. control; ${ }^{\#} P<0.05$, ${ }^{\# \#} P<0.01,{ }^{\# \# \#} P<0.001$ vs. hypoxia. a-d One-way ANOVA followed by Tukey's post hoc analysis

formation, and inflammatory cell infiltration, which were prevented by $\mathrm{KLX}-\mathrm{H}$ pretreatment. The protein levels of cleaved caspase-1, GSDMD, and N-terminal GSDMD (N-GSDMD) were markedly elevated in $\mathrm{MI}$, and these effects were significantly reversed by $\mathrm{KLX}-\mathrm{H}$ (Fig. 2c-f). Caspase-1 activation causes the transformation of IL-1 $\beta$ and IL-18 into their active forms, which in turn triggers further myocardial damage in mice subjected to MI. The release of cytokines into the blood is a hallmark of pyroptosis [6]. Thus, we further examined the expression of these two pro-inflammatory cytokines. The MI-induced elevation of the protein levels of intracardiac IL-1 $\beta$ and IL-18 in the LV was significantly repressed by $\mathrm{KLX}-\mathrm{H}$ treatment compared with vehicle treatment (Fig. $2 g, h$ ). These results indicated that KLX suppresses pyroptosis in MI mice.

KLX attenuates the activation of the NLRP3 inflammasome upon $\mathrm{MI}$ in mouse hearts

The NLRP3 inflammasome, a pro-inflammatory protein complex, has been shown to be activated and to contribute to cell death via pyroptosis in a caspase-1-dependent manner [19]. The inhibition of the NLRP3 inflammasome offers cardioprotective benefits by reducing cardiomyocyte pyroptosis [10]. To determine whether the anti-pyroptotic effects of $\mathrm{KLX}$ are attributable to the suppression of the NLRP3 inflammasome, we measured the NLRP3 protein level. Consistent with a published study [20], our Western blot analysis showed that MI resulted in the upregulation of NLRP3 $24 \mathrm{~h}$ after MI, and similar expression patterns of proteins downstream of NLRP3, including mature IL-1 $\beta, N-G S D M D$, and GSDMD, were observed, supporting the notion that NLRP3 inflammasome activation initiates downstream effectors associated with pyroptosis (Fig. 3a, b). Moreover, KLX significantly inhibited the elevation of NLRP3 mRNA and protein levels induced by MI (Fig. 3c, d).

KLX inhibits pyroptosis in cardiomyocytes

Hypoxia is a critical event in the setting of $\mathrm{MI}$ and is well recognized to simulate in vivo ischemic conditions in cells [21, 22]. In addition, hypoxia has been shown to promote pyroptosis 
Ctrl LPS

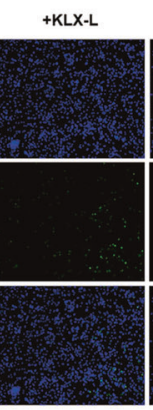

+KLX-M
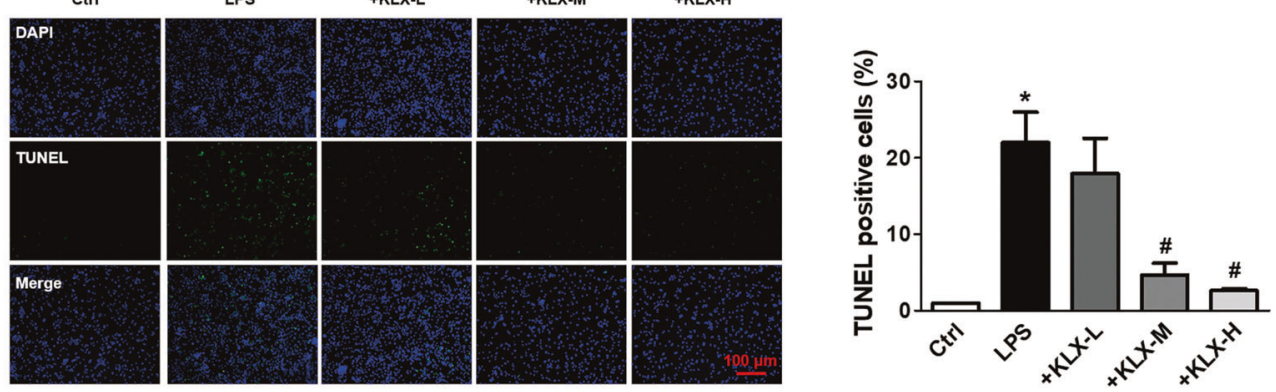

b
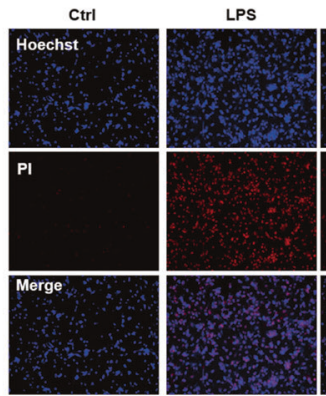

+KLX-L

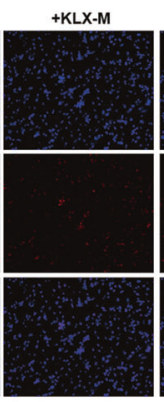

+KLX-H
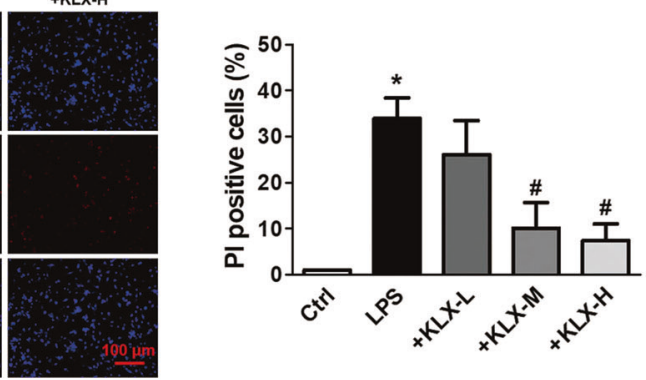

c
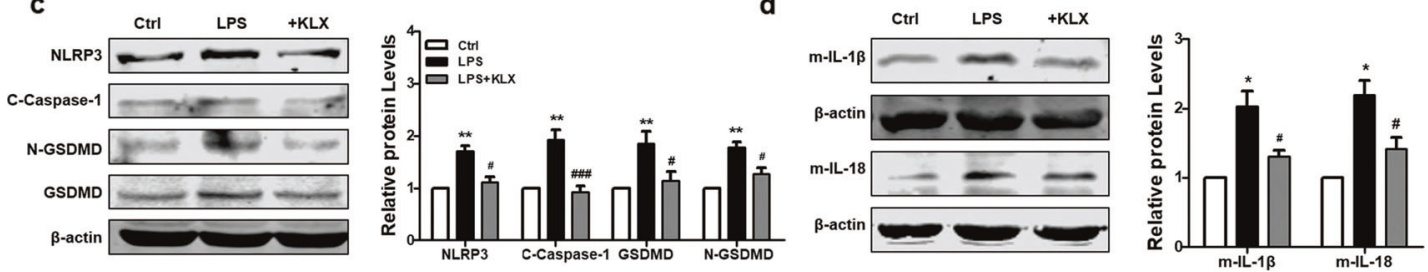

Fig. 5 Effects of KLX on LPS-mediated NLRP3 activation and pyroptosis in cardiomyocytes. a Representative images of TUNEL staining and the quantitative analysis of TUNEL-positive cardiomyocytes from each group. The NMVCs were pre-treated with different doses of KLX for $24 \mathrm{~h}$ and exposed to $1 \mu \mathrm{g} \cdot \mathrm{mL}^{-1}$ LPS for $12 \mathrm{~h}$. Scale bar $=100 \mu \mathrm{m} ; n=3$. b Representative images of Hoechst 33342/PI staining and quantitative analysis of PI-positive cardiomyocytes from each group. Scale bar $=100 \mu \mathrm{m} ; n=3$. c, d Western blot analysis of NLRP3 ( $n=5$ ), cleaved caspase$1(n=5)$, GSDMD $(n=5)$, N-GSDMD $(n=5)$, mature IL-1 $\beta(n=4)$, and IL-18 $(n=5)$ protein levels in NMVCs treated with $10 \mu M$ KLX. The data are presented as the mean \pm SEM. ${ }^{*} P<0.05,{ }^{* *} P<0.01$ vs. control; ${ }^{\#} P<0.05$, ${ }^{\# \# \# ~} P<0.001$ vs. LPS. a-d One-way ANOVA followed by Tukey's post hoc analysis

through NLRP3 inflammasome activation [12]. To determine whether KLX can prevent cardiomyocytes from pyroptosis, we cultured NMVCs under hypoxic conditions. Cell membrane rupture and chromosomal DNA fragmentation are morphological features of pyroptosis [23]. The numbers of TUNEL-positive cells and PIpositive cells were both significantly increased in the hypoxia group compared with the normoxia group, and treatment with 10 and $20 \mu \mathrm{M} \mathrm{KLX}$, but not $5 \mu \mathrm{M} \mathrm{KLX}$, significantly blunted these deleterious changes in cardiomyocytes (Fig. 4a, b). Moreover, hypoxia markedly elevated the protein expressions of NLRP3, cleaved caspase-1, GSDMD, N-GSDMD, IL-1 $\beta$, and IL-18, and KLX reversed these alterations (Fig. $4 c, d$ ). These results indicated that $\mathrm{KLX}$ protects against cardiomyocyte pyroptotic cell death in response to hypoxia.

Lipopolysaccharide (LPS) has been shown to cause cardiomyocyte pyroptosis and simulate inflammatory injury resembling $\mathrm{MI}$ [24]. To corroborate our in vitro observations, we investigated the effects of KLX on LPS-induced changes in NMVCs. LPS stimulation significantly induced cardiomyocyte pyroptosis, as indicated by the increased numbers of TUNEL- and PI-positive cells, which were remarkably suppressed by treatment with 10 and $20 \mu \mathrm{M} \mathrm{KLX}$ (Fig. 5a, b). In accordance with our observations under hypoxic conditions, LPS also elicited profound increases in the expression levels of NLRP3 and the pyroptosis-related proteins mature IL-1 $\beta$ and IL-18, and these increases were significantly abrogated by $10 \mu \mathrm{M} \mathrm{KLX}$ (Fig. 5c, d).

\section{DISCUSSION}

Here, we report the beneficial effects of a novel chemically modified monomeric compound, $\mathrm{KLX}$, on cardiac damage, its function in a mouse model of $\mathrm{MI}$ and the underlying mechanisms. Our results showed that KLX significantly reduces the infarct size and improves cardiac dysfunction induced by MI. Moreover, KLX significantly suppressed the expression of the NLRP3 inflammasome, the release of the pro-inflammatory cytokines IL-1 $\beta$ and IL18 and pyroptotic cell death in both an in vivo model of $\mathrm{MI}$ and an in vitro model of ischemic injury by hypoxia or LPS (Fig. 6).

Ml-induced cardiac ischemia remains one of the leading causes of mortality worldwide, despite the use of advanced therapeutic interventions and drugs. The major reason for myocardial injury upon $\mathrm{Ml}$ is cardiomyocyte death, which is also a therapeutic target for preventing $\mathrm{MI}$ and subsequent consequences. However, commonly used drugs are limited by their therapeutic efficacy and side effects [25-27]. Accumulating evidence has shown that traditional Chinese medicine offers huge beneficial effects in the treatment of heart diseases [28, 29]. In the current study, we found that KLX had beneficial effects on reducing the myocardial infarct size and LDH release by affecting the NLRP3 inflammasome and pyroptosis.

Pyroptosis is a newly identified form of inflammatory cell death triggered by caspase-1, and it has been reported to be casually linked to a variety of diseases, particularly to cardiomyocyte loss following $\mathrm{MI}$ and ischemia/reperfusion [9, 30, 31]. GSDMD acts as 


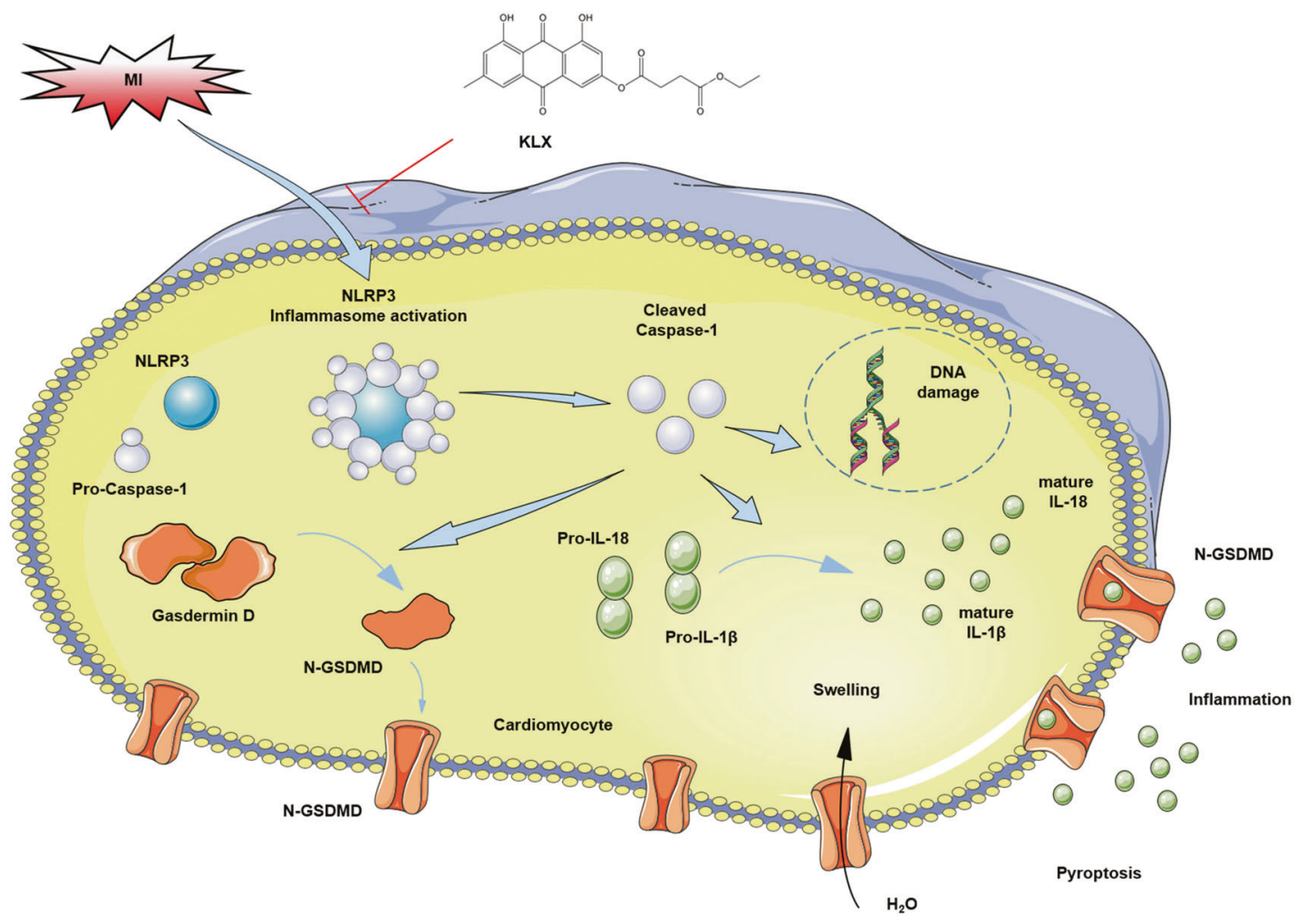

Fig. 6 Underlying mechanisms of the protective effect of KLX against myocardial infarction

the executor of pyroptosis by producing an N-terminal fragment, which oligomerizes in the cell wall and causes pyroptotic cell death by forming membrane pores [5]. Moreover, the inhibition of caspase- 1 decreases the infarct size and improves cardiac function by attenuating cardiomyocyte pyroptosis [11]. Our study showed that KLX significantly suppressed the cardiac production of several pyroptosis-related proteins, including GSDMD, N-GSDMD, and cleaved caspase-1. The morphological features of pyroptosis are nuclear condensation, DNA fragmentation, and the destruction of cell membrane integrity. As a result, the intracellular ion balance is destroyed, cell swells, and excess inflammatory reaction is triggered after the release of the cellular contents [32]. In agreement with this view, our results indicated that $\mathrm{Ml}$ induces pyroptosis in the heart, as evidenced by increased DNA fragmentation and mitochondrial swelling and cell membrane rupture, and notably, these detrimental alterations were markedly reversed by $\mathrm{KLX}$ treatment. These results suggest that the protective effects of KLX on the ischemic heart can likely be ascribed to the attenuation of pyroptosis.

MI initiates an intensive inflammatory response that is essential for cardiac wound healing, but it can also aggravate cardiac damage and dysfunction [33]. NLRP3 is a member of the inflammasome, which is a large multimeric protein complex, and the NLRP3 inflammasome can readily be activated upon tissue injury [34]. MI triggers NLRP3 inflammasome activation, leading to the conversion of IL-1 $\beta$ and IL-18 to their active mature forms in a caspase-1-dependent manner [35]. IL-1 $\beta$ and IL-18 are early and strong pro-inflammatory cytokines that expand the infarct size and drive cardiac dysfunction [35]. The pharmacological inhibition of IL-1 $\beta$ and IL-18 with a neutralizing antibody significantly reduces myocardial infarct size and ameliorates cardiac dysfunction in MI mice $[36,37]$. Additionally, the NLRP3 inflammasome promotes ischemic myocardial injury by causing pyroptosis via activating caspase-1 [8], whereas the inhibition of the NLRP3 inflammasome reduces the myocardial infarct size and preserves normal cardiac function post $\mathrm{MI}[20,33,38]$. In line with the reported finding that NLRP3 is activated in acute MI [38], our results showed that NLRP3 inflammasome expression was increased, reaching a significant difference at $24 \mathrm{~h}$ in the infarcted myocardium of mice. Furthermore, KLX significantly attenuated the elevation of NLRP3, IL-1 $\beta$, and IL-18 protein levels. H\&E staining analysis showed reduced inflammatory cells in MI mice treated with KLX. Collectively, these data suggest that KLX reduces cardiac damage by attenuating the pro-inflammatory response and reducing subsequent pyroptosis.

Hypoxia and LPS are potent stimuli for cardiomyocyte injury and the secretion of inflammatory factors, which can in part mimic the conditions of cardiac damage in the setting of $\mathrm{MI}[22,39]$. Recent studies have found that hypoxia and LPS induce cell death by activating the NLRP3 inflammasome and pyroptosis in cardiomyocytes $[12,22]$. Our results showed that the levels of IL$1 \beta, I L-18$, NLRP3 and pyroptosis-related proteins were diminished in NMVCs treated with KLX. Our TUNEL and Hoechst 33342/PI staining results further demonstrated that KLX improved DNA damage and cell membrane rupture of cardiomyocytes induced by hypoxia or LPS treatment, indicating that it suppressed pyroptotic cell death in cardiomyocytes. There was no significant difference in the protective effect between the $10 \mu \mathrm{M}$ and $20 \mu \mathrm{M}$ $\mathrm{KLX}$ groups, indicating that the cardioprotective effect of KLX was efficacious at $10 \mu \mathrm{M}$ in our model.

In conclusion, our study provides the first evidence that KLX exerts myocardial protection by inhibiting NLRP3 inflammasome activation and subsequent pyroptosis in $\mathrm{Ml}$ hearts and in cardiomyocytes treated with hypoxia or LPS as a cellular model of $\mathrm{MI}$ injury. Our results suggest that KLX merits more rigorous and detailed studies regarding its potential for the treatment of MI. In this regard, it is known that inflammasomes and caspase-1/ pyroptosis are important parts of the innate immune system. It has also been suggested that agents targeting innate immunity may exert acute cardioprotective effects [40]. Our finding that KLX acts 
on the components of innate immunity (i.e., inflammasomes and caspase-1/pyroptosis) encourages further studies on this process.

\section{ACKNOWLEDGEMENTS}

This work was supported in part by the National Natural Science Foundation of China (grants number: 81730012, 81603105, 81773733, and 81573425), the Scientific Research Starting Foundation for Returned Overseas Chinese Scholars of Heilongjiang Province, the Heilongjiang Natural Science Foundation (grants number: H2016010), and the China Postdoctoral Science Foundation (2017M621309).

\section{AUTHOR CONTRIBUTIONS}

BFY, WX, WJD, NW, and YB designed the present study; YB, XL, PP, XLH, STY, and YNL performed the experiments; JHW conducted the synthesis of KLX; YB, XL, PP, and $\mathrm{XL}$ analyzed the data; YB, WJD, and BFY wrote the paper; WJD and NW revised paper.

\section{ADDITIONAL INFORMATION}

The online version of this article (https://doi.org/10.1038/s41401-019-0307-8) contains supplementary material, which is available to authorized users.

Competing interests: The authors declare no competing interests.

\section{REFERENCES}

1. Cohn JN, Ferrari R, Sharpe N. Cardiac remodeling-concepts and clinical implications: a consensus paper from an international forum on cardiac remodeling. Behalf of an International Forum on Cardiac Remodeling. J Am Coll Cardiol. 2000;35:569-82.

2. Task Force on the management of ST-segment elevation acute myocardial infarction of the European Society of Cardiology (ESC), Steg PG, James SK, Atar D, Badano LP, Blomstrom-Lundqvist C, et al. ESC Guidelines for the management of acute myocardial infarction in patients presenting with ST-segment elevation. Eur Heart J. 2012;33:2569-619.

3. Wang PP, Zhang YJ, Xie T, Sun J, Wang XD. MiR-223 promotes cardiomyocyte apoptosis by inhibiting Foxo3a expression. Eur Rev Med Pharmacol Sci. 2018;22:6119-26.

4. Olivier E, Dutot M, Regazzetti A, Laprevote O, Rat P. 25-Hydroxycholesterol induces both P2X7-dependent pyroptosis and caspase-dependent apoptosis in human skin model: New insights into degenerative pathways. Chem Phys Lipids. 2017;207:171-8

5. Liu X, Zhang Z, Ruan J, Pan Y, Magupalli VG, Wu H, et al. Inflammasome-activated gasdermin $D$ causes pyroptosis by forming membrane pores. Nature. 2016;535:153-8.

6. Bergsbaken T, Fink SL, Cookson BT. Pyroptosis: host cell death and inflammation. Nat Rev Microbiol. 2009;7:99-109.

7. Mezzaroma E, Toldo S, Farkas D, Seropian IM, Van Tassell BW, Salloum FN, et al. The inflammasome promotes adverse cardiac remodeling following acute myocardial infarction in the mouse. Proc Natl Acad Sci USA. 2011;108: 19725-30.

8. Toldo S, Abbate A. The NLRP3 inflammasome in acute myocardial infarction. Nat Rev Cardiol. 2018;15:203-14.

9. Takahashi M. NLRP3 inflammasome as a novel player in myocardial infarction. Int Heart J. 2014;55:101-5.

10. Lei Q, Yi T, Chen C. NF-kappaB-Gasdermin D (GSDMD) axis couples oxidative stress and NACHT, LRR and PYD domains-containing protein 3 (NLRP3) inflammasome-mediated cardiomyocyte pyroptosis following myocardial infarction. Med Sci Monit. 2018;24:6044-52.

11. Audia JP, Yang XM, Crockett ES, Housley N, Haq EU, O'Donnell K, et al. Caspase-1 inhibition by VX-765 administered at reperfusion in $\mathrm{P} 2 \mathrm{Y} 12$ receptor antagonisttreated rats provides long-term reduction in myocardial infarct size and preservation of ventricular function. Basic Res Cardiol. 2018;113:32.

12. Chen A, Chen Z, Xia Y, Lu D, Yang X, Sun A, et al. Liraglutide attenuates NLRP3 inflammasome-dependent pyroptosis via regulating SIRT1/NOX4/ROS pathway in H9c2 cells. Biochem Biophys Res Commun. 2018;499:267-72.

13. Yu Y, Liu H, Yang D, He F, Yuan Y, Guo J, et al. Aloe-Emodin attenuates myocardial infarction and apoptosis via up-regulating MiR-133 expression. Pharmacol Res. 2019;146:104315.

14. Huang J, Li X, Liu P, Wang J, Li H. Emodin protects H9c2 cells against hypoxiainduced injury via regulation of miR-26a/survivin and the JAK1/STAT3 pathway. J Cell Biochem. 2019; https://doi.org/10.1002/jcb.28385. (In press).

15. Yang $Y$, Jiang Z, Zhuge D. Emodin attenuates lipopolysaccharide-induced injury via down-regulation of miR-223 in H9c2 cells. Int Heart J. 2019;60:436-43.
16. Liu J, Li Y, Tang Y, Cheng J, Wang J, Li J, et al. Rhein protects the myocardiac cells against hypoxia/reoxygention-induced injury by suppressing GSK3beta activity. Phytomedicine. 2018;51:1-6.

17. Bai $Y$, Su Z, Sun H, Zhao W, Chen X, Hang P, et al. Aloe-emodin relieves high-fat diet induced QT prolongation via MiR-1 inhibition and IK1 up-regulation in rats. Cell Physiol Biochem. 2017;43:1961-73.

18. Bock-Marquette I, Saxena A, White MD, Dimaio JM, Srivastava D. Thymosin beta4 activates integrin-linked kinase and promotes cardiac cell migration, survival and cardiac repair. Nature. 2004;432:466-72.

19. Mezzaroma E, Toldo S, Abbate A. Role of NLRP3 (cryopyrin) in acute myocardial infarction. Cardiovasc Res. 2013;99:225-6.

20. Toldo S, Marchetti C, Mauro AG, Chojnacki J, Mezzaroma E, Carbone S, et al. Inhibition of the NLRP3 inflammasome limits the inflammatory injury following myocardial ischemia-reperfusion in the mouse. Int J Cardiol. 2016;209:215-20.

21. Zhang Y, Jiao L, Sun L, Li Y, Gao Y, Xu C, et al. LncRNA ZFAS1 as a SERCA2a inhibitor to cause intracellular $\mathrm{Ca}^{2+}$ overload and contractile dysfunction in a mouse model of myocardial infarction. Circ Res. 2018;122:1354-68.

22. Zhou Z, Wang Z, Guan Q, Qiu F, Li Y, Liu Z, et al. PEDF inhibits the activation of NLRP3 inflammasome in hypoxia cardiomyocytes through PEDF receptor/phospholipase A2. Int J Mol Sci. 2016;17, pii: E2064.

23. Shi J, Gao W, Shao F. Pyroptosis: gasdermin-mediated programmed necrotic cell death. Trends Biochem Sci. 2017;42:245-54.

24. Chen J, Wang B, Lai J, Braunstein Z, He M, Ruan G, et al. Trimetazidine attenuates cardiac dysfunction in endotoxemia and sepsis by promoting neutrophil migration. Front Immunol. 2018;9:2015.

25. Arnold SV, Spertus JA, Masoudi FA, Daugherty SL, Maddox TM, Li Y, et al. Beyond medication prescription as performance measures: optimal secondary prevention medication dosing after acute myocardial infarction. J Am Coll Cardiol. 2013;62:1791-801.

26. Love JN, Enlow B, Howell JM, Klein-Schwartz W, Litovitz TL. Electrocardiographic changes associated with beta-blocker toxicity. Ann Emerg Med. 2002;40:603-10.

27. Boggild M. Intracerebral haemorrhage after dermal nitrate application. BMJ. 1992;305:1000.

28. Wu B, Lin J. Dihydromyricetin protects against diabetic cardiomyopathy in streptozotocin-induced diabetic mice. Biomed Res Int. 2017;2017:3764370.

29. Pan C, Lou L, Huo Y, Singh G, Chen M, Zhang D, et al. Salvianolic acid B and tanshinone IIA attenuate myocardial ischemia injury in mice by NO production through multiple pathways. Ther Adv Cardiovasc Dis. 2011;5:99-111.

30. Nazir S, Gadi I, Al-Dabet MM, Elwakiel A, Kohli S, Ghosh S, et al. Cytoprotective activated protein $C$ averts Nlrp3 inflammasome-induced ischemia-reperfusion injury via mTORC1 inhibition. Blood. 2017;130:2664-77.

31. Dolunay A, Senol SP, Temiz-Resitoglu M, Guden DS, Sari AN, Sahan-Firat S, et al. Inhibition of NLRP3 inflammasome prevents LPS-induced inflammatory hyperalgesia in mice: contribution of NF-kappaB, caspase-1/11, ASC, NOX, and NOS isoforms. Inflammation. 2017;40:366-86.

32. Galluzzi L, Vitale I, Abrams JM, Alnemri ES, Baehrecke EH, Blagosklonny MV, et al. Molecular definitions of cell death subroutines: recommendations of the Nomenclature Committee on Cell Death 2012. Cell Death Differ. 2012;19:107-20.

33. van Hout GP, Bosch L, Ellenbroek GH, de Haan JJ, van Solinge WW, Cooper MA, et al. The selective NLRP3-inflammasome inhibitor MCC950 reduces infarct size and preserves cardiac function in a pig model of myocardial infarction. Eur Heart J. 2017;38:828-36.

34. Bullon P, Cano-Garcia FJ, Alcocer-Gomez E, Varela-Lopez A, Roman-Malo L, RuizSalmeron RJ, et al. Could NLRP3-inflammasome be a cardiovascular risk biomarker in acute myocardial infarction patients? Antioxid Redox Signal. 2017;27:269-75.

35. Deten A, Volz HC, Briest W, Zimmer HG. Cardiac cytokine expression is upregulated in the acute phase after myocardial infarction. Experimental studies in rats. Cardiovasc Res. 2002;55:329-40.

36. Abbate A, Van Tassell BW, Seropian IM, Toldo S, Robati R, Varma A, et al. Interleukin-1 beta modulation using a genetically engineered antibody prevents adverse cardiac remodelling following acute myocardial infarction in the mouse. Eur J Heart Fail. 2010;12:319-22.

37. Venkatachalam K, Prabhu SD, Reddy VS, Boylston WH, Valente AJ, Chandrasekar B. Neutralization of interleukin-18 ameliorates ischemia/reperfusion-induced myocardial injury. J Biol Chem. 2009;284:7853-65.

38. Jong WM, Leemans JC, Weber NC, Juffermans NP, Schultz MJ, Hollmann MW, et al. Nlrp3 plays no role in acute cardiac infarction due to low cardiac expression. Int J Cardiol. 2014;177:41-3.

39. Yin J, Wang Y, Hu H, Li X, Xue $M$, Cheng W, et al. P2X7 receptor inhibition attenuated sympathetic nerve sprouting after myocardial infarction via the NLRP3/IL-1beta pathway. J Cell Mol Med. 2017;21:2695-710.

40. Zuurbier CJ, Abbate A, Cabrera-Fuentes HA, Cohen MV, Collino M, De Kleijn DPV, et al. Innate immunity as a target for acute cardioprotection. Cardiovasc Res. 2019;115:1131-42. 